\title{
Heterogénea producción científica estudiantil en Perú: análisis regional y propuestas
}

Carlos J. Toro-Huamanchumo, Jessica Meza-Liviapoma, Cender U. Quispe-Juli, José E. Fernández-Chinguel, Junior S. Torres-Román

El pregrado de medicina es la etapa ideal para formar al estudiante en una cultura de investigación. La clave es asegurar una educación médica de calidad desde sus inicios y cosechar los frutos más tarde [1]. En diversos países de Latinoamérica existen las sociedades científicas de estudiantes de medicina (SOCEM), agrupaciones que buscan fomentar un ambiente científico, académico y de servicio durante el pregrado $[1,2]$.

En Perú, la Sociedad Científica Médico Estudiantil Peruana (SOCIMEP) es la entidad que actualmente abarca 37 SOCEM de ámbito nacional, las cuales a su vez se agrupan en consejerías regionales. Existen tres consejerías, correspondientes a la región norte, centro y sur del país, cada una de las cuales congrega diferentes sociedades. Además, en cada una se realizan actividades de manera independiente (pero no restringida) a las demás regiones. Uno de los criterios para esta distribución es la proximidad entre las ciudades donde se ubican [2]. No obstante, dado que las oportunidades y limitaciones para investigar varían de acuerdo a la universidad [3], la producción científica entre las SOCEM difiere considerablemente [4]. Por ello, uno de los indicadores para evaluar la importancia y el papel de estas consejerías debe ser la producción científica por regiones.

Con el objetivo de conocer la producción científica regional de las sociedades científicas estudiantiles del Perú, se efectuó una búsqueda de las publicaciones realizadas por las SOCEM durante los años 2002-2014. Se incluyó todo tipo de publicación en una revista indizada y al menos un autor debía ser estudiante de medicina, miembro de una SOCEM en el momento de envío del artículo a publicación. Se revisó la base de datos Medline y Google Scholar. Para la búsqueda y confirmación de las publicaciones se usó una estrategia descrita previamente [4].
Se hallaron un total de 369 publicaciones, de las cuales el $20,6 \%$, $87 \%$ y $6,8 \%$ correspondieron a publicaciones del norte, centro y sur, respectivamente. La suma de los porcentajes supera el $100 \%$, pues se encontraron casos de publicaciones con colaboración institucional entre sociedades $(n=53)$. En general, se observa una producción con tendencia al crecimiento, pero con marcada diferencia interregional. La región centro tuvo más publicaciones en Scopus, ISI o Medline $(n=113)$, mientras que el norte y sur tuvieron 10/76 y $9 / 25$, respectivamente.

Son dos las situaciones que merecen atención: por un lado, no todas las SOCEM cuentan con producción científica (en el año 2014 sólo publicaron 19 sociedades), y por otro, el número de publicaciones difiere considerablemente entre las regiones, concentrándose la mayor parte en el centro del país. La primera circunstancia tiene su base en los obstáculos ya conocidos para publicar en pregrado: no contar con asesores y ausencia de incentivo para realizar investigación [3,5]. A esto se agrega la deficiente formación universitaria en materia de redacción y publicación científica, probablemente relacionada con la baja frecuencia de publicación de los docentes de investigación del país [6]. Por todo esto, es importante la formación de redes de colaboración, incluyendo la participación activa de los asesores, como herramienta para solucionar el centralismo de la investigación en Perú [7]. SOCIMEP también debe fortalecer sus estrategias vigentes [5] e innovar otras. Una de las más recientes es la creación del Comité de Publicaciones Científicas, cuyo objetivo principal es capacitar, asesorar y reconocer la producción científica estudiantil. En general, esta situación debe evaluarse prospectivamente $y$, en caso de ser necesario, plantear nuevos criterios de distribución regional. Es un trabajo en equipo en el que se deben sumar esfuerzos, restar limitaciones y generar un efecto multiplicador.
Sociedad Científica de Estudiantes de Medicina Veritas, SCIEMVE; Facultad de Medicina Humana; Universidad de San Martín de Porres; Lima (C.J. Toro-Huamanchumo, J.E. Fernández-Chinguel). Sociedad Científica de Estudiantes de Medicina de la Universidad Nacional de Piura, SOCIEM-UNP; Facultad de Medicina Humana; Universidad Nacional de Piura; Piura (J. MezaLiviapoma). Sociedad Científica de Estudiantes de Medicina Agustinos, SOCIEMA; Facultad de Medicina Humana; Universidad Nacional San Agustín; Arequipa (C.U. Quispe-Juli). Sociedad Científica de Estudiantes de Medicina de Ica, SOCEMI; Facultad de Medicina Daniel Álcides Carrión; Universidad Nacional San Luis Gonzaga; Ica, Perú (J.S. TorresRomán).

Correspondencia:

Carlos Jesús Toro Huamanchumo. Sociedad Científica de Estudiantes de Medicina Veritas (SCIEMVE). El Muelle Ltes. 5, 6 y 7. Las Delicias. Chiclayo 14001. Lambayeque, Perú.

E-mail:

toro2993@hotmail.com

(c) 2015 FEM 
Bibliografía

1. Taype-Rondán A, Palma-Gutiérrez E, Palacios-Quintana M, Carbajal-Castro C, Ponce-Torres C. Producción científica estudiantil en Latinoamérica: un análisis de las revistas médicas de habla hispana indizadas en SciELO, 2011. FEM 2014; 17: $171-7$.

2. Toro-Huamanchumo CJ. ¿Por qué pertenecer a sociedades científicas en el pregrado de medicina? Rev Cuerpo Méd HNAAA 2014; 7: 43-4.

3. Mayta-Tristán P, Cartagena-Klein R, Pereyra-Elías R, Portillo A, Rodríguez-Morales AJ. Apreciación de estudiantes de medicina latinoamericanos sobre la capacitación universitaria en investigación científica. Rev Med Chile 2013; 141: 716-22.
4. Taype-Rondán A, Bazán-Ruiz S, Valladares-Garrido D. Producción científica de las sociedades científicas de estudiantes de medicina del Perú, 2002-2012. CIMEL 2013; 18: 23-9.

5. Taype-Rondán A. Huaccho-Rojas J, Guzmán L. Sociedades científicas de estudiantes de medicina en el Perú: situación actual y perspectivas futuras. CIMEL 2011; 16: 90-5.

6. Pereyra-Elías R, Huaccho-Rojas J, Taype-Rondán A, Mejía CR, Mayta-Tristán P. Publicación y factores asociados en docentes universitarios de investigación científica de escuelas de medicina del Perú. Rev Peru Med Exp Salud Publica 2014; 31: 424-30.

7. Huamaní C, Mayta-Tristán P. Producción científica peruana en medicina y redes de colaboración, análisis del Science Citation Index 2000-2009. Rev Peru Med Exp Salud Publica 2010; $27: 315-25$ 